\title{
POLYMYALGIA RHEUMATICA AND GIANT CELL ARTERITIS
}

\author{
Gillian Pountain, Brian Hazleman
}

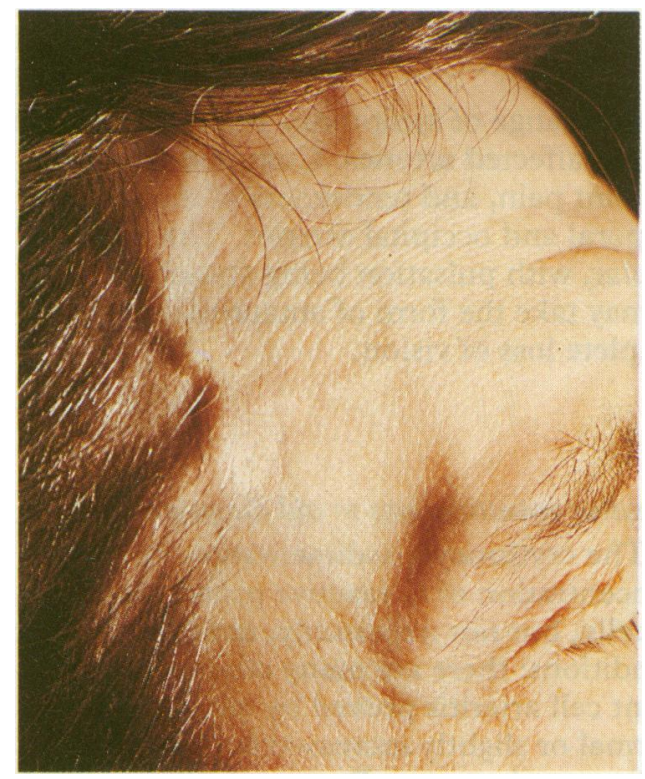

Swollen temporal artery of patient with giant cell arteritis.

\section{Aetiology}

\section{Aetiology. of polymyalgia rhoumatica and giant cell arteritis}

- There is often a distinct prodromal event resembling influenza, but results of viral studies are negative

- Lymphocytes in arteritic lesions express the $T$ cell phenotype, and the CD4 subset predominates

- Frequency of HLA-DR4 is increased

\section{Epidemiology of giant cell arteritis}

- Peak incidence at ages 60-75

- Sex distribution of 3 women to 1 man

- Annual incidence 18/100 000 people aged over $50^{*}$

- Prevalence 2230/100 000 people aged over $50 *$

- Most reports from northern Europe and northern United States; mainly affects white people, but can occur worldwide

- Familial aggregation has been reported, suggesting genetic association

*Diagnosis confirmed by biopsy
In recent years giant cell arteritis and polymyalgia rheumatica have increasingly been considered as closely related conditions. The two syndromes form a spectrum of disease and affect the same types of patient. The conditions may occur independently or may occur in the same patient, either together or separated in time.

Polymyalgia rheumatica is a clinical syndrome of middle aged and elderly patients and is characterised by:

- Pain and stiffness in the neck and the shoulder and pelvic girdles

- Systemic features such as low grade fever, fatigue, and weight loss

- An increased erythrocyte sedimentation rate

- A dramatic response to small doses of corticosteroids.

Polymyalgia rheumatica-At present it is impossible to define the underlying pathological abnormality in polymyalgia rheumatica, and several different mechanisms may be responsible for a largely similar pattern of pain.

Giant cell arteritis is limited to vessels with an internal elastic component. Both humoral and cellular immunological mechanisms have been implicated in its development, and the latter seem to be more important.

Giant cell arteritis affects white people almost exclusively, but it has been reported in black Americans. In all studies both conditions are very rare under the age of 50 years. Reports from Olmsted County, Minnesota, and Gothenburg, Sweden, have shown that the incidence is increasing in women but not in men. About half of patients with giant cell arteritis have symptoms of polymyalgia rheumatica, whereas $15-50 \%$ of patients with polymyalgia rheumatica have giant cell arteritis. Problems with case definition and ascertainment complicate epidemiological observations. 


\section{Differential diagnosis of polymyalgia rheumatica}

Myeloma

Neoplastic disease

Joint disease

Osteoarthritis, particularly of cervical spine

Rheumatoid arthritis

Connective tissue disease

Muscle disease

Polymyositis

Myopathy

Infections
Diagnosis may be difficult, and polymyalgia rheumatica is diagnosed by exclusion.

\section{Polymyalgia rheumatica}

Symptoms are usually bilateral and symmetrical. The predominant feature is usually stiffness that is particularly severe after rest and may prevent a patient getting out of bed. Affected structures feel tender, and shoulder movement may be restricted if diagnosis is delayed. Muscular pain is often diffuse and is accentuated by movement. Muscle strength is unimpaired, but the pain makes interpretation of muscle testing difficult. Persistent synovitis is uncommon and suggests an alternative diagnosis such as rheumatoid arthritis.

\section{Giant cell arteritis}

This condition causes a wide range of symptoms, but most patients have clinical features related to affected arteries. Common features include fatigue, headaches, joint pain, and tenderness of the scalp, particularly around the temporal and occipital arteries. The arteries are thickened, tender, and nodular, with pulsation being absent or reduced. Visual symptoms may take the form of amaurosis fugax, diplopia, and partial or complete loss of vision.

\section{Investigations}

\section{Investigations at presentation of polymyalgia rheumatica and giant cell arteritis}

Polymyalgia rheumatica

- Erythrocyte sedimentation rate

- Acute phase proteins

- Full blood count

- Biochemical profile

- Protein electrophoresis

- Bence Jones proteins.

- Thyroid function

- Chest radiograph

- Rheumatoid factor

- Muscle enzymes (if indicated)

\section{Baseline investigations to aid diagnosis}

The erythrocyte sedimentation rate and concentration of $\mathrm{C}$ reactive protein are usually, but not necessarily, raised in the two conditions: there are many case reports of giant cell arteritis proved by biopsy with normal or slightly increased erythrocyte sedimentation rates. Repeated measurements may show raised erythrocyte sedimentation rates after an initial normal value.

Patients may have normocytic anaemia, and the concentration of hepatic alkaline phosphatase is often raised. These features, together with the clinical picture of weight loss, may resemble those of occult malignancy.

If polymyalgia rheumatica is suspected other conditions must be excluded by appropriate investigations.

\section{Biopsy for giant cell arteritis}

- Perform biopsy if diagnosis is in doubt, particularly if systemic symptoms predominate

- Biopsy is most useful within 24 hours of starting treatment, but do not delay treatment for sake of biopsy

- A negative result does not exclude giant cell arteritis

- A positive result helps to prevent later doubts about diagnosis, particularly if treatment causes complications

\section{Biopsy}

Some patients with polymyalgia rheumatica but without symptoms of giant cell arteritis have positive results from temporal artery biopsies. Thus, all suspected cases of polymyalgia rheumatica or giant cell arteritis might benefit from temporal artery biopsy. However, the problems of obtaining biopsies rapidly make this impractical. The choice of patients for biopsy depends on local circumstances, but a pragmatic policy would be to select only patients with suspected giant cell arteritis (not those with obvious clinical features). Patients with pure polymyalgia rheumatica would need to be monitored carefully for development of clinical giant cell arteritis.

A third of patients with signs and symptoms of cranial arteritis may have negative temporal artery biopsies, which may be due to the localised involvement of arteries in the head and neck. After one week of corticosteroid treatment the chance of obtaining a positive biopsy result is only $10 \%$, so biopsy is worth while only in the first few days of treatment. However, treatment for suspected giant cell arteritis should not be delayed simply to allow a biopsy to be carried out. 


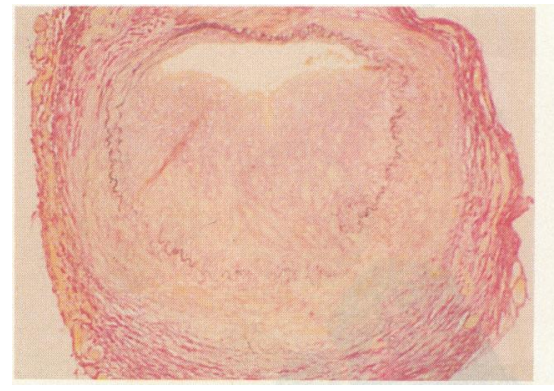

Histological appearance of artery affected by giant cell arteritis: decreased lumen and disruption of internal elastic lamina (left); inflammatory cell infiltrate (right).

\section{Histology of giant cell arteritis}

Giant cell arteritis is one of the most distinctive pathological disorders. In the acute phase the histological changes are characteristic, but several arterial diseases with different clinical syndromes may also have a granulomatous histological appearance. Typical changes include a giant cell infiltrate and disruption of the media and internal elastic lamina. The intima is thickened and oedematous, and the lumen is restricted. Pathologists should be aware of the wide range of histological changes that occur as part of normal aging and must not interpret these as evidence of healed arteritis. The histological changes of healed arteritis include medial chronic inflammation with a growth of new blood vessels, focal medial scarring, and intimal fibrosis.

\section{Treatment}

\section{Treatment of polymyalgia rheumatica and giant cell arteritis*}

\section{Polymyalgia rheumatica \\ Initial dose-Prednisolone $10-20 \mathrm{mg}$ daily for one month \\ Reduce dose by $2.5 \mathrm{mg}$ every 2-4 weeks until dose is $10 \mathrm{mg}$, then by $1 \mathrm{mg}$ every $4-6$ weeks (or until symptoms return) \\ Maintenance dose of about $10 \mathrm{mg}$ by six months after start of treatment and 5-7.5 mg by one year. Most patients require treatment for 3-4 years but withdrawal after two years is worth attempting}

\section{Giant cell arteritis}

Initial dose-Prednisolone 20-40 mg daily for eight weeks. Patients with ocular symptoms may need up to $80 \mathrm{mg}$ daily

Reduce dose by $5 \mathrm{mg}$ every 3-4 weeks until dose is $10 \mathrm{mg}$ daily, then as for polymyalgia rheumatica

Maintenance dose of about $3 \mathrm{mg}$ daily may be required

-Recurrence of symptoms requires an increase in prednisolone dose

\section{Diagnosing relapse of polymyalgia rheumatica and giant cell arteritis}

- Principally a clinical diagnosis

- Erythrocyte sedimentation rate and concentration of $C$ reactive protein are often not raised in relapses; when they are raised another cause is often found

\section{Risks of side effects from corticosteroid treatment}

- Increased risk with high initial doses (>30 mg) of prednisolone, maintenance doses of $10 \mathrm{mg}$, and high cumulative dosages - Maintenance doses of $5 \mathrm{mg}$ prednisolone are relatively safe

1 Skingle SJ, Crisp AJ. Increased bone density in patients on steroids with etidronate. Lancet 1994;344:543-4.
Treatment with corticosteroids is mandatory for giant cell arteritis to prevent vascular complications, particularly blindness, as well as for rapidly relieving symptoms: before corticosteroids were used the reported prevalence of blindness was $30-60 \%$. Corticosteroids are usually also used for polymyalgia rheumatica because of the possibility of blindness.

Most patients require three to four years of treatment with corticosteroids, and many need continued treatment with small doses. If a patient needs high doses of corticosteroid, azathioprine or methotrexate can be used to reduce the required dose by their corticosteroid sparing effect, but regular monitoring of blood count and testing of liver function are required.

\section{Relapses}

Relapses are most likely in the first 18 months of treatment, but they can occur after apparently successful treatment when corticosteroids have been discontinued. At present there is no way of predicting which patients are most at risk. Diagnosis of relapse should be made on clinical features since the erythrocyte sedimentation rate and concentration of $\mathrm{C}$ reactive protein are often not raised during relapses or may be increased due to other causes.

During relapses the dose of prednisolone should be increased to the dose given before relapse or more, depending on the severity of symptoms.

\section{Complications}

Patients are at risk of the usual side effects of corticosteroids. The reported incidence of side effects ranges from $20 \%$ to $50 \%$. In one study side effects were significantly more common with a starting dose of prednisolone of $30 \mathrm{mg}$ or more or a mean maintenance dose of over $5 \mathrm{mg}$. Side effects can be minimised by using low doses of prednisolone whenever possible and giving corticosteroid sparing drugs such as azathioprine and methotrexate when necessary.

In elderly people corticosteroid treatment carries the risk of increasing osteoporosis. For women, disodium etidronate with calcium carbonate is the most effective prophylaxis. It increases the spinal bone mineral density of patients taking corticosteroids by about $4 \%$ after one year and by $4 \cdot 8 \%$ after two years. ${ }^{1}$

Gillian Pountain is consultant rheumatologist at Hinchingbrooke Hospital, Huntingdon, and Brian Hazleman is consultant rheumatologist at Addenbrooke's Hospital, Cambridge.

The ABC of Rheumatology is edited by Michael L Snaith, senior lecturer in rheumatology at the Institute for Bone and Joint Medicine, University of Sheffield. 